\title{
Urgences
}

\section{Dialogues avec l'organiste Jean-Guy Proulx}

\section{Jean-Claude Brochu}

Numéro 29, octobre 1990

Éclats d'œuvre

URI : https://id.erudit.org/iderudit/025613ar

DOI : https://doi.org/10.7202/025613ar

Aller au sommaire du numéro

Éditeur(s)

Urgences

ISSN

0226-9554 (imprimé)

1927-3924 (numérique)

Découvrir la revue

Citer ce document

Brochu, J.-C. (1990). Dialogues avec l'organiste Jean-Guy Proulx. Urgences, (29),

77-92. https://doi.org/10.7202/025613ar d'utilisation que vous pouvez consulter en ligne.

https://apropos.erudit.org/fr/usagers/politique-dutilisation/ 


\section{Dialogues avec l'organiste Jean-Guy Proulx Jean-Claude Brochu}

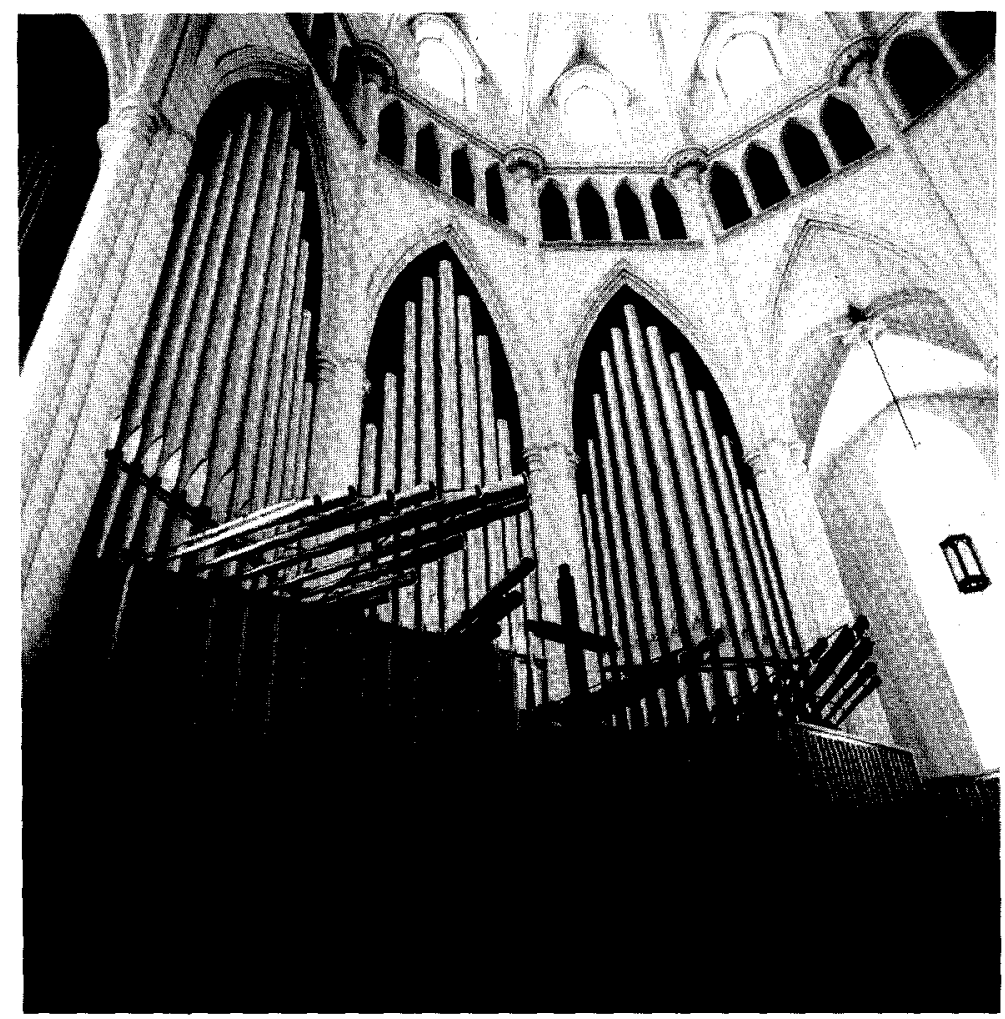

Grand orgue de la Cathédrale Saint-Germain de Rimouski 
Jean-Guy Proulx et Jean-Claude Brochu, coauteurs du texte qui suit, sont, le premier, organiste titulaire à la cathédrale Saint-Germain de Rimouski et professeur au Conservatoire, le second, chargé de cours en français à l'Université de Montréal et professeur de littérature québécoise au collège Édouard-Montpetit à Longueuil.

J.-C. B. Jean-Guy Proulx, vous êtes organiste, et l'un des meilleurs de votre génération d'après les critiques qui comptent, vous avez gravé trois disques, vous enseignez; où figure le vrai mystère de la musique pour vous? Quel est celui que vous cherchez à approfondir depuis plus de vingt ans?

J.-G.P. Je retiens les deux points de votre question: le vrai mystère de la musique et celui que je cherche à approfondir. Mais en guise de préliminaire, je voudrais d'abord parler du Beau en soi d'après Schönberg:

C'est à l'artiste pourtant que le Beau se révèle, mais sans qu'il l'ait recherché, puisqu'il ne tend que vers le Vrai.

Le Beau, si toutefois il existe, est insaisissable; celui-là seul le fait jaillir, qui possède la force de contemplation nécessaire à l'éveiller, et le créer chaque fois de nouveau, par la seule force de cette contemplation.

Le Beau n'existe qu'à partir du moment où les profanes, ceux qui ne produisent pas, commencent à en ressentir l'absence.

Dans ce Beau transcendantal et platonicien, il va de soi que je recherche le Vrai (qui est son autre nom). Comme interprète, je suis en présence d'un langage, celui de l'orgue, d'un instrument et d'un répertoire très étendu dans le temps. Donc, je dois le dire tout de suite, les notions même de Beau et de Vrai filtrent à travers le Beau et le Vrai des prédécesseurs. C'est ainsi qu'il existe une sorte de nomenclature du Beau: nous parlerons de chefs-d'œuvre, d'œuvres accessibles, d'œuvres clés et d'œuvres de transition. Mais constamment au-delà de ces diverses « déclinaisons* du Beau léguées par une tradition, il faut viser l'essentiel, soit le message du compositeur dans sa quintessence. Je mettrais d'ailleurs au premier plan, avant même l'interprétation subjective que je peux faire du Beau et du Vrai selon lui, la situation de l'artiste.

Pour retourner sur la première phrase de Schönberg et sur ce Beau qui n'advient que par le Vrai, j'ajouterais que l'interprète doit appuyer son exécution sur une très solide 
formation, sur une connaissance profonde des styles, des époques et des cuvres. À cet examen minutieux doit se greffer encore, à mon sens, un synopsis des arts en général, de la peinture, de la littérature, de la politique et de la société dans laquelle ces cuvres ont été créées. Pour moi, œuvre et contexte sont totalement indissociables.

Quant à l'étude fouillée des styles, il ne s'agit pas du style pour le style... Prenons l'exemple de la musique baroque, que l'on peut jouer sur des instruments anciens, je crois que c'est maintenant tout à fait fondamental - ayant fait des recherches stylistiques à ce sujet - de jouer cette musique d'orgue sur les instruments pour lesquels elle a été écrite. Question d'authenticité encore une fois. Et j'ajouterais qu'un interprète doit à tout le moins connaítre l'existence d'un tel débat avant d'interpréter les œuvres de cette époque. Je ne prétends pas qu'on doive toujours réunir les conditions idéales pour jouer, parce qu'à ce compte-là, il faut en convenir, nous serions très limités. Notre siècle nous permet présentement de jouer toutes les musiques sur à peu près tous les instruments. Bien sûr, idéalement, je dois connaître les caractéristiques de l'orgue de la Renaissance de Frescobaldi, de l'orgue baroque du temps de Bach, les caractéristiques de l'orgue romantique au temps de Liszt ou de Franck, ainsi que celles des orgues symphonique et néo-classique de Messiaen ou de Jean Langlais. Ces savoirs à propos des instruments, des styles, du répertoire forment un tout où le Vrai et le Beau s'articulent autour d'une connaissance - ma connaissance subjective en tant qu'interprète. Il s'agit d'un moteur pour tout artiste dans son ascension vers la vérité. Vérité subjective, passée au tamis de ma sensibilité et nourrie par une expérience, une recherche et des études comparatives dont le résultat sera ma personnalité. Mais le substrat de ma personnalité reste ce qu'aura voulu livrer le compositeur.

Je veux encore souligner que ce Beau et ce Vrai, à un premier degré, celui de la feuille écrite que je ne peux que décrypter par le médium d'un instrument, ces mêmes notes pourraient vouloir dire des choses différentes selon qu'elles ont été écrites du temps de Sweelinck à la Renaissance, du temps de Bach, ou du temps de Louis Vierne. Donc cette graphique, ou Auffuhrungspraxis, si l'on retourne au manuscrit, nous aide également à saisir les intentions de chacun des compositeurs. Partant de ces intentions, je fais moi-même 
80

ma propre interprétation puisque la musique ne devient vivante qu'au moment où elle est interprétée. Une feuille de musique, je peux la lire, je peux l'entendre, je peux l'imaginer, la concevoir dans ma tête, mais ce ne sera jamais comme la musique vraie, entendue et ressentie au moment d'une interprétation publique, ou au moment de la restitution sur disque d'une interprétation qui a déjà été arrêtée dans le temps. Il y a là un phénomène extrêmement vivant. Dans ce Vrai et ce Beau, dans cette recherche d'un absolu, je veux encore mentionner que l'auteur a livré pour les générations futures un texte à interpréter de toute urgence (n'applaudissez pas !).

Mais je dois aussi me rendre compte de l'influence de ma culture sur les critères que j'adopte pour juger du vrai. Comparons deux interprétations des Suites de Bach: la première gravée par Karajan dans les années soixante avec la Philharmonique de Berlin et l'autre jouée sur des instruments d'époque par John Eliot Gardiner et The Baroque Soloists. Nous voyons là, pour une même musique, deux interprétations différentes. Je crois cependant que Karajan était aussi vrai que Gardiner. Ce sont tout simplement le point de vue (chef d'orchestre) et le médium (orchestre et instruments) qui donnent à ces deux musiques des personnalités distinctes. Comme organiste, je ressens exactement la même chose.

Je ne suis qu'un interprète, cela dit sans fausse modestie, je disparais derrière les volontés, derrière même le message du compositeur. Ce qui ne veut pas dire que je ne suis pas original. Je vais essayer de saisir le plus pur de ce messagelà, mais en le rendant vivant, en n'étant pas figé, en n'ayant pas peur d'y aller de ma propre imagination et d'aller dans ce que le compositeur ne pouvait même pas écrire, dans ce qui commence à être beau, "le bon goût " comme le définissait François Couperin dans L'art de toucher le clavecin. Finalement, on peut savoir toutes les règles, elles peuvent toutes nous être dictées mais ce sera encore le bon goût qui fera qu'une interprétation correspondra ou non au désir d'un compositeur. Le sens de la musique, en un mot. Même si la musique demeure toujours souveraine, exactement comme dans le cas de l'histoire, on peut dire: tant vaut l'interprète, tant vaut la musique.

J.-C. B. Quelques lectures récentes m'ont fourni au moins un point de rencontre à propos de la musique. Ainsi, si je 
prolonge la pensée d'Allan Bloom dans L'Ame désarmée, la musique serait l'art le plus éducatif en ce sens qu'elle s'attache à réconcilier la part dionysiaque avec la part apollinienne en l'homme. Elle élève le désir jusqu'à la transcendance du Bien et du Bon. Les plaisirs musicaux instruiraient sur les tâches de la vie, l'agréable informerait le meilleur. Psychanalytiquement, il s'agirait d'une sorte de compromis entre le "ça" et l"idéal du moi».

Dans le même élan, Vladimir Jankélévitch (Le Nocturne) suggère - mezza-voce - l'idée de sublimation: sorte de coincidentia oppositorum où le corps se transporte (pas question de l'exclure) dans le lieu de l'esprit pour s'y confondre avec ("de bas en haut et de l'étroit au large comme les puissances biologiques de la végétation »). La musique ennoblirait la confusion. Elle ritualise le passage entre ce qui finit et ce qui commence, entre - d'après ses propres mots "un Déjà-plus et un Pas-encore». Déjà plus corps et pas tout à fait esprit.

Et Julien Green, dans le plus récent tome de son Journal, de ramener l'opposition inclusive par la ferveur et la sexualité mêlées, la science et la fougue, et surtout de conclure: "... la musique est le vrai langage universel. Elle dit à chacun ce qu'il a besoin d'entendre et chacun étant un être différent se trouve uni aux autres dans la même admiration parce qu'il croit que cette grande voix souveraine lui parle de la même façon qu'aux autres. » La musique extériorise l'immobilité d'un cri intérieur à la fois trop humain, déjà plus humain et pas encore au-delà. Elle fonde une "communauté dans les notes" en débrouillant le sens perdu du Bien commun.

En tant que professeur et praticien, que pensez-vous de cette fonction pédagogique, lato sensu, de la musique?

J.-G.P. Revenons d'abord à Schönberg, si vous le voulez bien: "Le Beau n'existe qu'à partir du moment où les profanes, ceux qui ne produisent pas, commencent à en ressentir l'absence. « Quand je joue, la musique n'existe qu'au moment où elle est ressentie et entendue par d'autres. Certains continueront à penser que cette musique peut rester hermétique, peut rester enveloppée dans un idéal de faire placé si haut, si élevé, que, pour certains, ils ne pourront de leur vie le livrer. Je ne suis pas de ceux-là. J'ai franchi la rampe avec humilité et simplicité pour livrer au public ce que 
82

je croyais être un idéal qui me quittait - sans m'avoir jamais appartenu en propre, dont j’étais dépositaire - et qui était susceptible de devenir l'idéal de quelqu'un d'autre. Je dois dire que l'expérience musicale que j'ai depuis plus de vingt ans maintenant m'amène, de par les contacts que j'ai eus avec les divers publics à travers la centaine de récitals que j'ai pu donner, à idéaliser peut-être, mais en même temps à me rendre compte que le public qui m'entend jouer quitte le concert plus beau intérieurement qu'il n'y est venu. Je souhaiterais atteindre toujours le raptus romain tel que décrit par Stefan Zweig: * l'envol d'un esprit au-dessus de luimême *. Il y a un état de grâce établi entre l'interprète et l'auditeur qui le pousse à s'avancer vers ce que je suis en train de créer au moment où il le reçoit. En ce moment précis l'auditeur actualise dans sa propre vie ce qu'il entend et il espère secrètement la prolongation de cette beauté dans le quotidien. Par cette plénitude, en somme, il sort de l'instant et accède à la durée. Un commentaire qui me fut déjà fait dans ce sens :

Ce que vous venez de réaliser, il y a probablement cent mille personnes qui voudraient le faire avec cette méme perfection. Et vous le voyant faire, je me sentais en communion directe, et je vous donnais ce que je n'arrivais pas à faire, et je prenais ce que vous faisiez, et il devenait mien.

Donc, il y a un phénomène d'inconscient collectif, si je puis dire, une sorte de * ça *.

Comment se * fabrique * un tel état? S’il n'est pas tout entier donné... Je bâtis un programme de musique un peu sur le principe d'une harmonie imitative: à l'image peut-être d'un repas avec entrée, plat principal et dessert, ce dernier résumera l'ensemble tout en restant en soi quelque chose. Je vois la composition d'un programme comme ayant une introduction, ce qui, règle générale, existe dans la structure même du programme musical, la structure de l'ordonnance des pièces que je propose. Ainsi, quand je commence par une partita de Pachelbel ou un grand dialogue de Marchand, je veux toucher l'orgue, je veux prendre le public, je veux aller le chercher pour le mettre en situation. Je veux lui dire: * Vous êtes là; voilà l'instrument que vous allez entendre et voilà le lieu dans lequel nous allons nous réunir, dans lequel nous allons communiquer. * Et puis viennent des choses plus 
délicates. Viennent des moments plus fins, plus tendus, plus complexes peut-être, dans lesquels je vais insérer des chorals, des préludes et fugues de Bach, dans lesquels je vais insérer des mouvements lents - peut-être de symphonies.

Qu'est-ce que j'entends par état de grâce? C'est ce moment unique où tout à coup il $\mathrm{y}$ a correspondance parfaite entre le lieu, l'instrument, la musique, les âmes de l'exécutant et de ceux qui écoutent; où il y a communion dans un absolu qui se fait rare, mais qui se recherche et qui peut se créer. Donc voilà un moment plus mezza-voce, plus en douceur, où chacun peut se laisser pénétrer, sans comprendre très souvent. Et ce sont souvent les meilleurs moments, où on est là, envahi par la musique. Elle peut être douce comme puissante, mais à travers les œuvres des compositeurs, il y a toujours des éléments plus propices à ce climat, et je dirais que ce seront souvent les mouvements lents: ce seront les cantilènes, ce seront les mélopées qui se déploieront, et ici je pense au larghetto de la Cinquième Symphonie de Vierne que j'affectionne plus particulièrement et où, pendant quinze minutes, je développe une mélodie en variations, mais toujours sur ce même thème, mezza-voce, qui va chercher, je crois, l'âme de chacun dans ce qu'elle comporte de beauté duelle: apaisement et torture de la vérité.

J.-C. B. Pascal matérialisait l'art littéraire par les concepts de présence et d'absence. La chose littéraire, et par analogie la chose musicale, commence à exister, quitte la linéarité pour la tridimensionnalité, quand la beauté d'un signifiant tranche sur "les mots de la tribu» (Mallarmé), quand la plénitude musicale est vécue comme un manque... La musique nous devient intime après coup, dans la déception du constat suivant: le monde ne nous a pas accompagné dans nos transports, c'est nous qui avons bougé. Schönberg encore.

J.-G. P. Et je dirais, pour renchérir sur vos migrations (rires) et pour clore ce programme imaginaire que nous bâtissons ensemble: nous avons besoin de nous extérioriser.

Nous avons besoin de sortir de nos frontières; nous avons besoin d'aller plus loin que nos limites et, rendus là, généralement, c'est un feu d'artifice. Je dirais que c'est une cascade, je dirais que c'est la lumière magnifiée. Je pense à cette œuvre de Jeanne Landry qui me fut dédiée et qui s'appelle Orah (mot hébreu pour désigner la lumière), où elle 
a voulu magnifier la lumière dans toutes ses facettes et dans sa brillance, sa transparence, à travers le médium de la musique et de l'orgue. Voilà en somme ce qu'un programme musical peut revêtir.

À propos de la fonction pédagogique, gauchissons le titre de Bloom et disons que nous sommes désarmés à un certain moment. La musique oriente une recherche intérieure, consciente ou inconsciente. Tout art se doit d'inspirer la vie, et je sais, par des commentaires du public, que quelques auditeurs se retrouvent et s'identifient à un message à travers une personne. Et je dirais même, par une pratique de plus de vingt ans comme organiste titulaire de la Cathédrale, qu'à certains moments, il y a véritablement ecclesia. Faire église, c'est bien sûr se réunir pour la prière dans un lieu donné, mais ce peut être aussi dans le cadre d'un concert, par la musique, faire l'unité autour d'un Bien commun. Les barrières disparaissent et la musique nous dit, sotto voce, ce qu'il faut aimer.

J.-C. B. Une grande part des études consacrées aux arts se déplace, pour parler d'une certaine façon, de l'autre côté de la rampe: la peinture réside davantage dans l'œil du spectateur, et en littérature, on s'intéresse de plus en plus aux ramifications biologiques de l'acte de lecture, pour ne citer que ces deux exemples. Cette réaffirmation générale de la subjectivité ne doit pas vous laisser indifférent puisque, je le sais, vous êtes très sensible à la réception profane (qui n'est pas celle des critiques et des spécialistes) de la musique - et ce, surtout en récital. Quelle chimie souhaitez-vous catalyser? En d'autres mots, idéalement, pour qui jouez-vous?

J.-G. P. D'abord la chimie (rires). Elle passe par le répertoire. Je cherche donc, à travers les âges et les époques, ce que l'on peut tirer de plus éloquent. Deux situations peuvent se présenter à moi : je vais en récital, dans une série où le thème est imposé. Par exemple, l'intégrale des symphonies de Louis Vierne à laquelle j’ai participé. Mon jeu se situait alors dans un contexte esthétique donné: un compositeur, une intégrale, des œuvres majeures, une symphonie. Déjà là, je m'adressais davantage à des spécialistes ou à des amateurs férus de ce type de musique. Il va de soi que la musique de Louis Vierne très fin XIX ${ }^{\mathbf{e}}$ et début XX' $\mathrm{XX}^{\mathrm{e}}$, très cathédrale, très Notre-Dame de Paris, convient à une esthétique et à une approche instrumentale et sonore proches par exemple, des opéras de 
Wagner, proches des grandes symphonies de Mahler, par la seule monumentalité des œuvres. Et si je veux rejoindre quelqu'un qui préfere la musique de Monteverdi (ses madrigaux), la musique de Gesualdo, la musique de Josquin des Prés, les miniaturistes, la musique de la Renaissance et même la musique baroque, je ne vise plus le même public. Il a d'autres exigences. Je ne jouerai pas de la même façon.

Dans une première situation, l'alchimie s'opère donc autour d'un thème. Par ailleurs, si je joue les miniaturistes de la Renaissance ou les grandes fresques baroques, j'aurai comme médium des instruments qui se rapprocheront des instruments de l'époque, plus petits, et pour lesquels j'utiliserai des registrations, des couleurs de jeux, de sonorités qui seront spécifiques. Et là, même si l'on doit idéalement tout connaître, tout cultiver et tout approfondir (!), il y a des spécialistes de certaines musiques. Dans mon cas, je dirais que je suis davantage spécialiste de la musique symphonique. Pourquoi le suis-je? C'est très simple. C'est que depuis vingt ans je suis titulaire du grand orgue de la cathédrale qui, au point de départ, n'était pas un instrument, disons, d'une grande originalité. Il avait ses qualités, il avait ses grands défauts. Et en même temps, il servait, en raison des lieux dans lesquels il sonnait, l'acoustique fort large, les hautes voûtes, la puissance, il servait un récital. Depuis sa restauration par la maison Guilbault-Thérien, en 1979, cet instrument, avec ses quatre claviers et ses mille cinq cents tuyaux, est devenu l'un des plus beaux au Québec selon, bien sû́r, les spécialistes et la critique. Je suis fort heureux de me retrouver presque quotidiennement au clavier de cet instrument, mais je dois dire que cette cohabitation a développé chez moi un répertoire approprié à ses vastes sonorités, aux voûtes de la cathédrale, ainsi qu'à la sensibilité que j’y ai acquise dans un rapport presque dialectique. C'est un peu l'instrument qui nous choisit et qui choisit, d'une certaine façon, le répertoire et le public, par ricochet.

Si l'on veut réaliser idéalement certaines musiques, nous allons dans un lieu précis, dans un lieu où la musique va sonner à son idéal précisément. Nous avons à Rimouski, par exemple, l'orgue de Saint-Pie-X, magnifique instrument à traction mécanique de vingt et un jeux, construit en 1967 par la maison Casavant et Frères. Un instrument de premier ordre. Voilà un orgue pour jouer merveilleusement la musique 
86

baroque, autant française qu'allemande, et où le répertoire romantique est cependant un peu moins bien servi. Pourquoi ? Parce que la luminosité des mixtures de Saint-Pie-X, l'articulation de ses jeux de fond, la verdeur de ses jeux d'anches en font un instrument idoine pour la musique baroque. L'abbé Antoine Bouchard est d'ailleurs venu y graver un disque. Et je suis persuadé que si j'avais été, il y a vingt ans, titulaire de cet instrument ou d'un orgue de chapelle à un clavier, un pédalier et sept ou huit jeux, plutôt que titulaire de la Cathédrale, mon répertoire ne serait pas orienté dans les voies qui sont actuellement les miennes.

Par contre, je ne veux pas me confiner uniquement à ce pourquoi un instrument a été idéalement conçu, je veux aussi en faire un instrument universel. Il faut bien admettre que l'orgue de la Cathédrale n'est pas l'instrument rêvé pour la musique de Bach. Et pourtant, j'en joue beaucoup. Pourquoi est-ce que j'y tiens tant? Le message musical de Bach est à ce point universel qu'il supporte, je crois, l'« investigation " de tous les médiums. Autrement dit, un orgue imparfait n'épuise pas la perfection de Bach. Devant l'imperfection d'un instrument - et pas un orgue n'est parfait - , je dois consentir à des compromis en m'inspirant du compositeur. Je veux permettre à la musique de vivre, de s'incarner même imparfaitement; sous prétexte de fausse authenticité, je ne me prive pas et ne vous prive pas d'un plaisir. Même avec un instrument inapte à servir tous les répertoires, je trouve malgré tout un vaste éventail de styles, depuis Buxtehude et Pachelbel. J'ai enregistré une suite de Pierre Du Mage, organiste du XVIII' siècle, je joue Couperin et d'Aquin. J'aborde très largement la musique française et, d'une manière privilégiée, plus près de nous, la musique symphonique de l'époque romantique, soit Franck avec ses douze pièces, Liszt - que ma formation en piano m'aide à soutenir - ou Mendelssohn. Beaucoup de compositeurs nous ont malheureusement oubliés: Beethoven, Chopin, Debussy... Si l'on ajoute les compositeurs du XX' siècle tels Vierne, Widor, Duruflé, Messiaen, Antoine Reboulot et Jeanne Landry, voilà reconstitué l'univers sonore dans lequel j'essaie d'établir cette sorte d'* harmonie des sphères " ptolémaique dont j’ai précédemment parié: heureuse combinaison des éléments que sont la musique, le compositeur, l'instrument, l'interprète, le public et le concert. 
J.-C. B. En ma qualité de simple mélomane, j’adopte souvent comme critère pour juger des interprètes leur aptitude (si nécessaire) à chanter ou jouer legato. Quelles sont les difficultés que vous rencontrez en montant une pièce? Quels sont les degrés à gravir avant d'accéder à ce fondu enchaîné? Vous savez, cette sensation que chaque note empiète un peu sur la suivante avant de la chasser - la musicalité, quoi. La console de l'orgue de la cathédrale Saint-Germain de Rimouski étant mobile, elle n'est pas fixée devant le buffet, $y$ a-t-il, par exemple, un délai (de traction) entre la commande et la réponse?

J.-G. P. Cette question m'amène à parler de la musicalité, qui, pour moi, avant la technique impeccable, demeure l'assise même de la musique. Passe toujours de jouer toutes les notes, mais encore faut-il de plus les rendre avec son cœur... L'interprétation ne relève jamais de la pure technique. Par un contact fréquent avec les musiciens d'une époque, il faut déceler le message à exprimer et développer en soi la sensibilité pour le restituer. Nous ne chantons pas la musique de Bach comme celle de Mozart, bien que Mozart ait vénéré Bach; de même pour Beethoven et Liszt. Il y a toujours une filiation. Toute pudeur disparaît dans la musique expressive, la musique avec le cœur sur la main, la musique don de soi.

Parce que la vie n'existe pas dans la bibliothèque, la partition ne s'anime que lorsque je la tire de son rayon pour la regarder; déjà elle s'actualise dans ma tête par la correspondance entre les notes et les sons, la formation d'accords et d'harmonies. Mais le souffle n'arrive qu'à l'exécution. La première approche revient à traquer le sens du manuscrit - aller au fond des notes - avant même d'apprendre la pièce. Sœur Pauline Charron, r.s.r., à Rimouski, m'a donné cette éducation première: quête d'authenticité qui suppose l'épreuve de la confrontation dialectique de ma perception avec celle des autres.

En quoi consiste la connaissance d'une œuvre? À passer d'abord la partition à la loupe. Ce travail, pour une symphonie de Louis Vierne par exemple, pièce dense et complexe s'il en est, m'a pris neuf mois. Au-delà de la première lecture des notes, j'ai dû l'assimiler. Passé l'assimilation, je mémorise les temps forts, les enchaînements, les points de chute, les élans et les tombées, l'envol, etc. Legato, oui, mais aussi 
88

staccato. Il faut jouer égal, et ce, en jouant chaque note détachée par rapport à la précédente et à la suivante, exactement pareille. L'oreille est alors sollicitée au maximum. Je dois avoir présent à l'esprit ce que je veux faire, et enregistrer ce que je suis en train de faire. Cette mémoire de la correspondance entre bonne note et fausse note doit passer par l'œil, par l'oreille et, bien sûr, par la main et les pieds, par le muscle enfin. Ce muscle, une fois entraîné, sert la musicalité, se détend: la main sera souple, le trait virtuose se fera sans aucun effort apparent, laissant place à une construction avec sa base (très solide) de compréhension de l'œuvre.

Et je reprends ici cette idée d'Antoine Reboulot, mon maître, qui me disait en parlant des cuvres que je jouais : * L'inspiration dans un concert peut passer à un moment donné, par contre il ne faut pas miser sur l'improvisation de cette inspiration, sur l'attente de la musicalité; elle est à construire ». Mon crescendo et mon decrescendo sont à construire, mes intentions musicales doivent être très réfléchies. Si à un moment précis je veux aller au-delà, ou en deçà, je pourrai, mais sans jamais me demander avant l'interprétation d'une pièce: «Qu'est-ce que je vais en faire? " Au contraire, toute la construction doit figurer dans ma tête dès la première note. Je dois savoir ce que je veux jouer. Je ne dois pas être interrompu par une difficulté technique. Je ne dois que chanter la musique, être disponible à elle. Et pour y être vraiment disponible, il a fallu qu'elle devienne un alter ego.

Le dernier point de votre question commande un détour historique. L'histoire de l'orgue remonte à l'Antiquité. Déjà avec Ctebios et son orgue hydraulique, avec Héron d'Alexandrie, déjà au cirque chez les Romains, nous connaissons l'orgue. Pérotin à Notre-Dame de Paris écrivait des organa pour soutenir le plain-chant de la foule. Et bien sûr à la Renaissance, à l'époque baroque, à l'époque classique, à l'époque romantique et maintenant au $\mathrm{XX}^{\mathbf{e}}$ siècle, se sont développées des esthétiques de l'orgue. Sans entrer dans les détails de facture de l'orgue, disons simplement qu'il s'agit d'un instrument à vent doté de tuyaux, clavier et pédalier. Avec un orgue à traction mécanique tel celui de Saint-Pie-X, au moment où je joue, mon doigt sur la touche actionne directement la soupape en-dessous du tuyau qui fait entrer l'air et qui le fait jouer. Donc, il y a instantanéité. 
Je dirais que le $\mathrm{XX}^{\mathrm{e}}$ siècle a apporté à l'orgue, par la traction électrique qui permet, par exemple, à la console de la Cathédrale d'être déplacée, une plus étroite communication entre l'organiste et son public - par opposition à l'organiste en tribune. (Par contre, la tribune facilite un son qui vient de plus loin.) Précisons encore une fois que la traction de l'orgue de Saint-Pie-X, en comparaison de celle de la Cathédrale, est plus immédiate et plus directe. J'ai donc dû développer une approche un peu différée à cause du courant électrique, mais en même temps cette approche est devenue instantanée. Pour paradoxal que cela puisse paraitre, malgré ce laps de temps, j'ai une communication instantanée avec l'orgue. Nous avons appris, l'orgue et moi, à répondre en même temps. Mes doigts vont chercher une fraction de seconde avant le son que je désire entendre et, finalement, la simultanéité de ma pensée et de ce que j'entends est absolue.

J.-C. B. De même que l'on assimile l'orchestre à une collectivité, on peut regarder l'orgue comme une miniature de ce microcosme: réduction d'une réduction. Parlez-nous de cet instrument.

J.-G.P. Ce côté "orchestre», qui constitue l'apport du XIX" siècle à la facture d'orgue, a entraîné une surmultiplication des jeux: on a voulu s'approprier à tort la puissance de l'orchestre. L'orgue est un petit univers en soi, et même un sanctuaire. Je songe en particulier ici à quelques phrases de Jean-Sébastien Bach:

Personne ne peut construire un orgue véritable sans un don spécial de Dieu, c'est tout autre chose que de construire une maison ou même un clavecin. II faut que l'âme du musicien soit enfermée dans les tuyaux pour que ceux-ci puissent parler et chanter. Si l'amour ne l'a pas construit, l'orgue ne vit jamais réellement.

Cette pensée de Bach atteste qu'il y a certainement des instruments sans grande personnalité. Et on le sent tout de suite. Pour l'orgue de la Cathédrale, restauré en 1979, le facteur a passé neuf mois à réharmoniser chacun des tuyaux afin d'en faire un tout homogène. Il lui faut teinter l'instrument selon des règles très strictes pour qu'il sonne ensuite avec sa personnalité propre. Et comme chacune des églises possède (quand l'église possède un orgue) un instrument différent, il n'y a pas deux orgues semblables. L'organiste 
90

doit savoir distinguer un instrument de tous les autres. C'est absolument extraordinaire de découvrir, quand quelqu'un d'autre touche l'orgue de la Cathédrale, comme je n'entends pas le même instrument. J'ai parfois l'impression que l'organiste a véritablement trouvé ce que l'orgue recèle de mieux à dire et, à d'autres occasions, j'ai plutôt le sentiment qu'il est passé à côté. Il a voulu registrer - parce que c'est le mot qu'on emploie pour le choix des jeux - oui, il a voulu registrer en prenant des recettes qui, sur papier, vont bien, mais il n'a pas pris la peine d'écouter. L'art d'un organiste, c'est l'art d'écouter. Il n'a donc pas suffisamment écouté et il est passé à côté de ce que l'orgue pouvait dire. Un orgue a toujours besoin d'être provoqué.

On me fait souvent la remarque suivante: «Vous devez avoir un sentiment de puissance en jouant sur cet instrument. " En effet, il y a un sentiment de puissance inhérent à la force (symphonique) que l'orgue peut produire. Ce sentiment de puissance arrive cependant bien loin derrière la grandeur et la majesté. Non pas la puissance triviale et temporelle, mais la grandeur et la majesté qui relèvent de l'ordre des transcendantaux, de ce qui, dans l'humain, va vers l'absolu, et dépasse notre faiblesse. Cet idéal n'est peut-être accessible qu'au contact de cet instrument... Un instrument enfoui dans les tribunes et souvent réservé au culte, un instrument assez mystérieux et encore méconnu.

À ce titre, nous avons à Rimouski un organisme très actif dans la promotion de l'orgue et de son répertoire, et qui célèbre cette année son vingtième anniversaire: les Amis de l'orgue. Voilà une chose absolument exceptionnelle pour notre région. Avec celle de Québec, nous sommes la plus ancienne société encore active. Nous accueillerons en juillet de l'an prochain un congrès international de la F.F.A.O. (Fédération francophone des amis de l'orgue). Ces amis de l'orgue se réunissent en Europe depuis une vingtaine d'années et ils ont décidé de venir au Québec l'an prochain. Pendant les huit jours où ils seront présents chez nous, ils iront à Montréal, à Québec et ils s'arrêteront une journée à Rimouski pour entendre l'orgue de la Cathédrale et celui de Saint-Pie-X. Une halte dans leur trajet est aussi prévue pour visiter l'orgue historique de Saint-Roch-des-Aulnaies de même que le très bel orgue de Saint-Pascal, un orgue classique français de la maison Casavant. C'est donc dire que 
Rimouski est une ville d'orgue. La classe d'orgue du Conservatoire et Radio-Canada, par l'enregistrement de nom. breuses émissions réseau, contribuent aussi à cette vie musicale rimouskoise.

J'y contribue moi-même avec beaucoup d'humilité et de ferveur en perpétuant la tradition des organistes titulaires du grand orgue de la Cathédrale. (Le titulaire est celui qui est responsable de l'orgue, de l'accord de l'orgue, de la musique et de l'organisation de la musique dans une église.) Par ailleurs, des disques ont été gravés à Rimouski. Moimême, en 1981, j’ai enregistré un premier disque qui s'intitule Jean-Guy Proulx aux grandes orgues de la cathédrale de Rimouski et sur lequel on retrouve des œuvres de Du Mage, de Franck, de Litaize et de Vierne. Je viens de faire, en 1988, un troisième disque qui s'appelle Musiques symphoniques pour grand orgue, le deuxième étant celui de l'Intégrale des six symphonies pour orgue de Louis Vierne, dans laquelle je jouais la cinquième. La maison REM, à Lyon en France, en assure la diffusion internationale. Je veux tout simplement par là souligner que, quand Jean-Guy Proulx est à Paris ou à Londres, ce n'est plus Jean-Guy Proulx, c'est Rimouski! Et la musique qu'on y joue est universelle, jouée sur un instrument de très haut niveau et digne de l'attention des mélomanes. Et ils sont nombreux. Des gens viennent ensuite à Rimouski et me disent: « Nous voulons entendre directement l'instrument que nous avons apprécié sur disque.»

J.-C. B. Qu'est-ce qui fait que l'on aime ou pas l'orgue?

J.-G. P. Cela tient à de nombreux facteurs: la capacité d'intérioriser, d'arrêter le temps, de fréquenter une église, un répertoire qui vient nous rejoindre sans mots, seulement avec des couleurs, des sons et des rêves réalisables.

\section{Discographie}

Jean-Guy Proulx aux grandes orgues de la Cathédrale de Rimouski. Du Mage, Franck, Vierne, Litaize; SISCOM, SC-02 102, 1981. (épuisé)

Louis Vierne, Intégrale des six symphonies, au grand orgue Saint-Jean-Baptiste de Montréal, deux coffrets de deux disques compacts: 
92

1 - Symphonie $n^{\circ} 1$ : Jacques Boucher; symphonie $n^{\circ} 2$ : Gaston Harel; symphonie $n^{\circ} 4$ : Denis Regnaud;

2 - Symphonie $n^{\circ} 3$ : Antoine Reboulot; symphonie $n^{\circ} 5$ : Jean-Guy Proulx; symphonie $n^{\circ} 6$ : Jacquelin Rochette; REM-11047-2, 1987.

Musiques symphoniques pour grand orgue. Jean-Guy Proulx, Cathédrale Saint-Germain de Rimouski, Qc, REM-311078XCD, 1988. 